Revue internationale P.M.E.

Économie et gestion de la petite et moyenne entreprise

\title{
La formation des dirigeants de PME : enquêtes et expériences françaises récentes
}

\section{Pierre-Laurent Bescos}

Volume 3, numéro 1, 1990

URI : https://id.erudit.org/iderudit/1007950ar

DOI : https://doi.org/10.7202/1007950ar

Aller au sommaire du numéro

Éditeur(s)

Presses de l'Université du Québec

ISSN

0776-5436 (imprimé)

1918-9699 (numérique)

Découvrir la revue

Citer cette note

Bescos, P.-L. (1990). La formation des dirigeants de PME : enquêtes et expériences françaises récentes. Revue internationale P.M.E., 3(1), 118-120.

https://doi.org/10.7202/1007950ar d'utilisation que vous pouvez consulter en ligne. 


\title{
La formation des dirigeants de PME : enquêtes et expériences françaises récentes
}

\author{
Pierre-Laurent BESCOS \\ École supérieure de commerce de Paris
}

La formation des dirigeants de PME fait l'objet, à l'heure actuelle, de beaucoup d'investigations en France, car deux constatations s'opposent.

Il y a tout d'abord la nécessité économique de disposer d'entreprises compétitives dans le cadre de la résorption du chômage et de l'ouverture complète des frontières entre les pays du Marché Commun (C.C.E.) en 1993. Les PME, du fait de leur souplesse, sont celles où les réserves de dynamisme et d'embauche sont les plus importantes. Le renouvellement de leurs dirigeants sera également un enjeu majeur dans les prochaines années. Tout ceci suppose des efforts importants de formation des dirigeants (ou des futurs dirigeants) de PME.

Mais il y a ensuite la difficulté de cerner et de répondre à la demande des dirigeants de PME, tant en ce qui concerne sa forme que son contenu. Il semble que les méthodes et les programmes proposés doivent innover, si l'on veut rencontrer les besoins de ce public. Une adaptation est nécessaire.

Dans le cadre d'une enquête effectuée par le Centre de Formation Continue du Groupe E.S.C.P., un après-midi a été organisé par l'auteur de ces notes sur le thème de «La formation des dirigeants de P.M.E., enjeu de la compétitivité», mardi, le 21 mars 1989, dans les locaux du Groupe E.S.C.P.

Les questions suivantes ont été traitées et débattues :

- Quels sont les besoins de formation au sein des P.M.E.?

- Quels sont les besoins propres à leurs dirigeants?

- Ces besoins sont-ils modifiés avec la perspective du Marché Unique Européen?

- Sous quelle forme et avec quel contenu doit-on y répondre?

- Comment financer cet éventuel besoin de formation? 
Les intervenants venaient d'horizons variés : dirigeants de P.M.E., formateurs du Groupe E.S.C.P., représentants du Ministère de l'Industrie et d'organismes de formation.

Les thèmes des interventions concernaient les résultats d'enquêtes très récentes effectuées par divers organismes sur le sujet ${ }^{1}$. Un débat a été organisé pour tirer les conséquences de ces constatations, en particulier sur les formations à mettre en place pour répondre aux aspirations des dirigeants de P.M.E. Les résultats des enquêtes et des discussions sont résumés ici.

\section{$1 \quad$ La perception des besoins}

La plupart des propriétaires-dirigeants interrogés reconnaissent la nécessité de se former et admettent ne pas y consacrer assez de temps. Ils sont très conscients de leurs lacunes. Mais un certain nombre de freins existent; par exemple, le temps disponible, l'emprise du court terme et l'inadaptation de l'offre aux besoins ou à la psychologie du chef d'entreprise.

Il faut également admettre que certains dirigeants ne sont pas toujours conscients de leurs besoins, les expriment de manière confuse ou sont peu réceptifs. Par contre, certains partenaires de la P.M.E., comme les banquiers ou les formateurs, ne peuvent que constater des lacunes importantes à combler au fur et à mesure des étapes de développement. Ceci doit être tempéré par la constatation d'une élévation régulière du niveau général de formation des dirigeants. Par ailleurs, certaines compétences du dirigeant ne peuvent être modifiées, telles les compétences sociales, le sens de l'action ou le goût du risque.

Ce que l'on peut affirmer, c'est que la demande ne s'exprime pas toujours, car beaucoup d'entreprises de cette taille ne perçoivent pas la formation comme un des éléments de base pour la réalisation de leurs objectifs.

Il y a donc toute une demande potentielle pour laquelle des voies nouvelles d'incitation à la formation doivent être trouvées. Ceci est d'autant plus difficile à faire que les besoins exprimés sont très divers sur le plan des contenus et des modes d'organisation. Il reste que cette demande est solvable, car l'aspect coût vient en dernière position lorsque l'on cherche à cerner les freins à l'expression des besoins.

1 Les divers auteurs des enquêtes sont les suivants : le Centre de Formation Permanente du Groupe E.S.C.P. (P.-L. Bescos, 1989), le Ministère de l'Industrie et de l'Aménagement du Territoire (S.E.S.S.I.), l'A.F.P.L.A.N.E. (J. Boudeville), l'I.F.A.C.E. (E. Bruneau) et H. Mahé de Boislandelle (1985). Pour les expériences, nous avons retenu principalement celle de Rhodanim (M. Devaux) et celles décrites par (Le Boterf et Chataigner, 1987). 


\section{Les domaines de la formation}

Les besoins exprimés, souvent par les dirigeants déjà les mieux formés, sont très divers. Tout d'abord, l'objectif d'une formation doit être principalement d'acquérir des connaissances utilisables par la suite, de s'ouvrir sur l'extérieur ou de se remettre en cause.

Ces chefs d'entreprises indiquent en majorité que la création d'un marché unique européen en 1993 entraîne de nouveaux besoins de formation pour euxmêmes, surtout en droit, en fiscalité et en langues étrangères. Mais il convient de remarquer que certaines P.M.E. attendent l'échéance pour s'adapter ou ne se sentent pas concernées.

En ce qui concerne les domaines de formation, on retrouve les priorités déjà indiquées, avec également d'autres souhaits concernant la stratégie, la communication, la micro-informatique et la gestion financière.

\section{Les modalités d'une organisation adaptée de la formation}

Nous avons voulu, tout d'abord, tester un certain nombre de formules existantes et d'innovations. La solution ayant la préférence des dirigeants concerne principalement la formation en alternance à l'extérieur de l'entreprise; par exemple, un jour par semaine de formation avec un cycle étalé dans le temps, si nécessaire. Mais d'autres formules sont également prisées : formation concentrée sur une courte période, rencontre-échange avec d'autres dirigeants.

Le formateur professionnel ou le conseiller d'entreprise semblent les intervenants les plus recherchés pour un stage de formation (et non pas un autre dirigeant de PME). Mais ceci semble fonction de la formule choisie. Dans un cycle de type rencontre-échange, la présence d'un dirigcant comme formateur est essentielle. L'apport de cadres dirigeants ayant pratiqué les outils de gestion au sein de grandes entreprises n'est pas à négliger également.

La constitution d'un réseau d'entraide ou de rencontre semble parfois un préalable pour déclencher des actions de formation vers les dirigeants peu sensibilisés. L'expérience de RHODANIM en est une bonne illustration. S'inspirant de pratiques québécoises, cette association a mis en place une structure légère afin de coordonner des clubs de patrons de P.M.E. donnant naissance à des groupes de formation (formation entre dirigeants ou avec appel à des animateurs professionnels, suivant les besoins progressivement décelés).

Mais le choix d'une formule doit tenir compte de la disponibilité du dirigeant pour la formation. Celle-ci est très limitée dans l'année (un mois, le plus souvent) et dans une journée (la préférence va au matin ou à la fin d'après-midi). 
Tout ceci entraîne un choix difficile dont les critères principaux sont liés, cependant, aux besoins ressentis. En effet, c'est la qualité du programme et la réputation de l'organisme de formation qui fondent la décision. Le critère coût ne vient qu'en troisième position.

Pour faire ce choix, le dirigeant s'informe. Mais il se renseigne le plus souvent d'une manière que l'on peut qualifier de passive, faute de temps : c'est la documentation reçue par l'entreprise qui est la source principale.

En définitive, ces enquêtes permettent d'éclairer quelque peu les contenus et les modes d'organisation des formations souhaitées par les dirigeants de P.M.E. Il est possible de remarquer que les besoins exprimés par les entreprises reflètent également les préoccupations des responsables de l'économie, en particulier à propos des nécessités de formations liées à l'ouverture des frontières.

C'est dire qu'une demande existe. Mais nous devons procéder comme ces chefs d'entreprises : bien connaître le marché de la formation des PME et faire en sorte de nous adapter en proposant des solutions innovantes. Il reste qu'il faut trouver les voies permettant de sensibiliser les dirigeants restant à l'écart des demandes exprimées. Cette quête semble indispensable si l'on veut atteindre une compétitivité plus grande des PME françaises.

\section{BIBLIOGRAPHIE}

Bescos, P.-L., (1989b), Laformation des dirigeants de P.M.E., enjeu de la compétitivité, Paris, Cahiers de recherche du Groupe E.S.C.P., Délégation à la Recherche.

Le Boterf, G. et. Chataigner, Y, (1987), La formation continue des dirigeants de P.M.E., comment innover?, Paris, La Documentation Française, série Recherche en formation continue, Délégation à la formation professionnelle, Ministère des Affaires Sociales et de l'Emploi.

Mahé de Boislandelle, H., (1985), «P.M.E. en démarrage : du décideur au gestionnaire», Enseignement et Gestion, nouvelle série n 36, p. 65-70.

S.E.S.S.I. (Service des Statistiques Industrielles), (1988), L'état des P.M.I., Paris, Ministère de l'Industrie et de l'Aménagement du Territoire, Direction Générale de l'Industrie, Délégation à la P.M.I. 